Supporting Information

\title{
Eliminating Heat Injury of Zeolite in Hemostasis via Thermal Conductivity of Graphene Sponge
}

Yuping Lianga , Congcong Xü ${ }^{a}$, Fang Liu ${ }^{b}$, Shiyu Du ${ }^{b}$, Guofeng Li ${ }^{a, c, *}$, Xing Wanga,c,*

${ }^{a}$ Beijing Laboratory of Biomedical Materials, Beijing University of Chemical Technology, Beijing 100029, P. R. China;

${ }^{\mathrm{b}}$ Department of Gastroenterology, China-Japan Friendship Hospital, Beijing 100029, P. R. China;

${ }^{c}$ Key laboratory of Biomedical Materials of Natural Macromolecules (Beijing University of Chemical Technology), Ministry of Education, Beijing 100029, P. R. China;

* Corresponding authors.

E-mail address: chase.1g@163.com (G. Li); wangxing@mail.buct.edu.cn (X. Wang) 


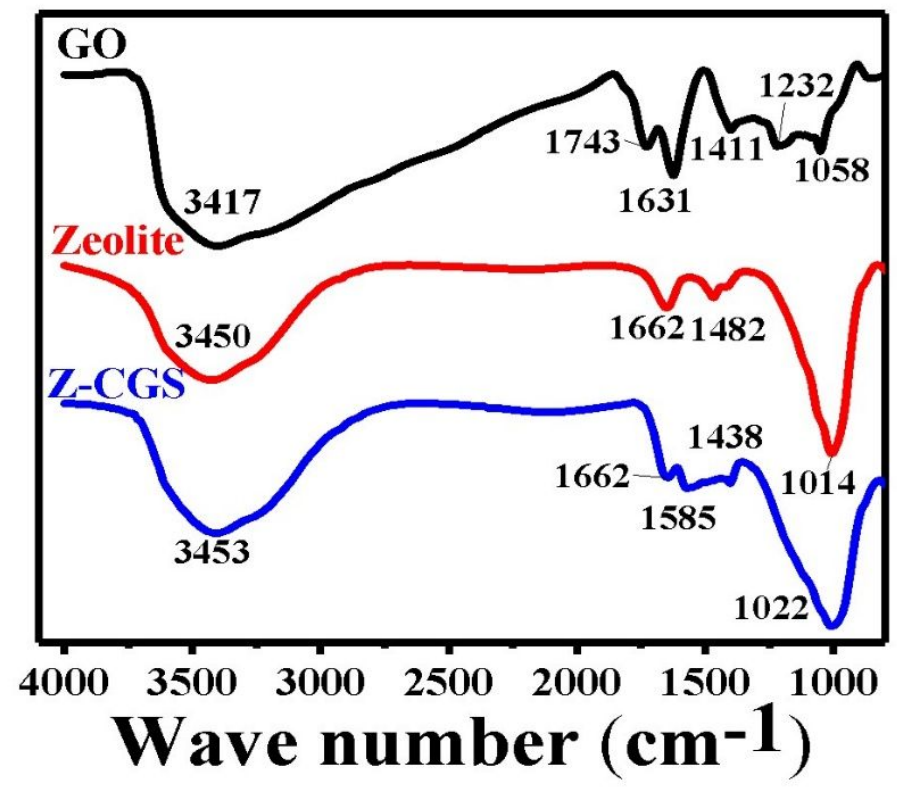

Figure S1. FT-IR spectra of GO, zeolite, and the Z-CGS 
$\mathbf{a}$

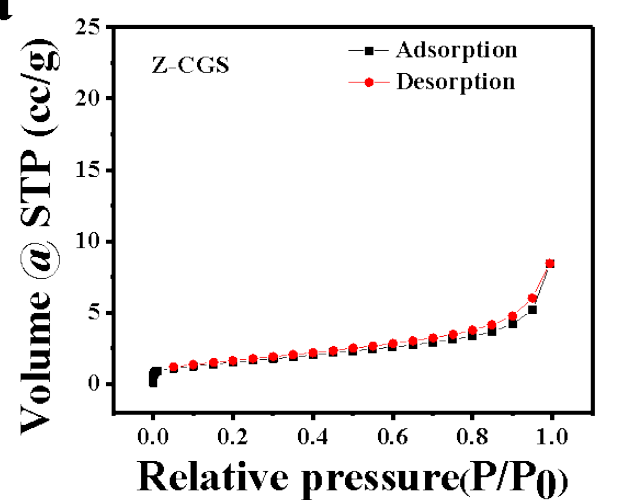

c

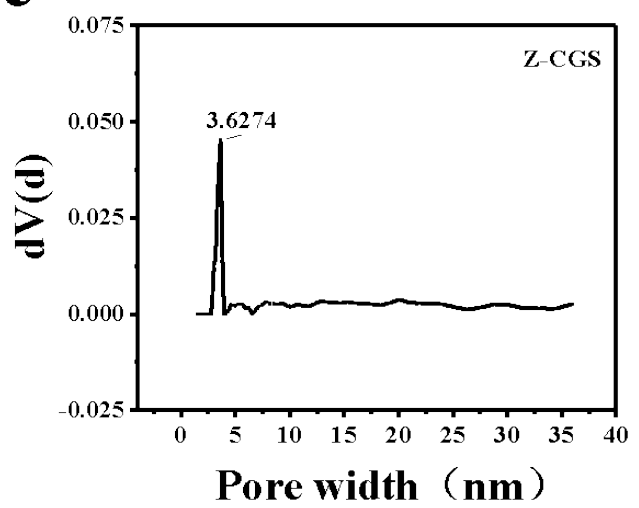

b

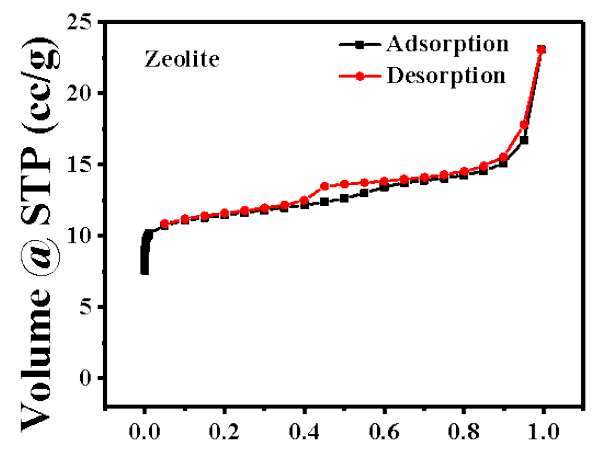

Relative pressure $\left(\mathbf{P} / \mathbf{P}_{\mathbf{0}}\right)$

d

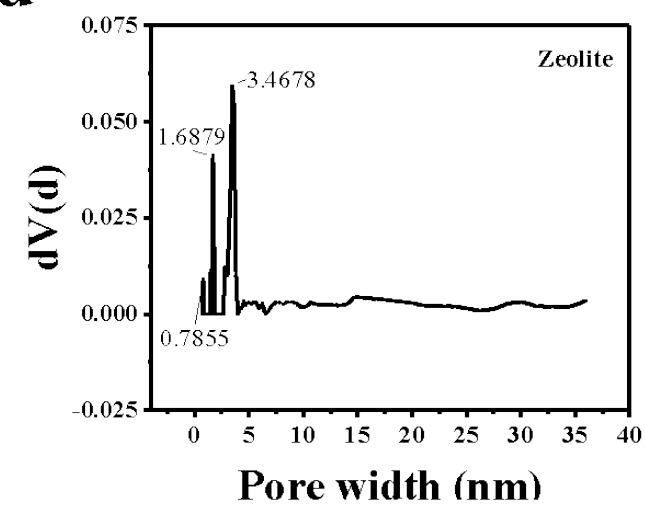

Figure S2. ( $a$ and b) The $\mathrm{N}_{2}$ adsorption-desorption isotherm and (c and d) the BJH pore size distribution of the Z-CGS and zeolite. 
a

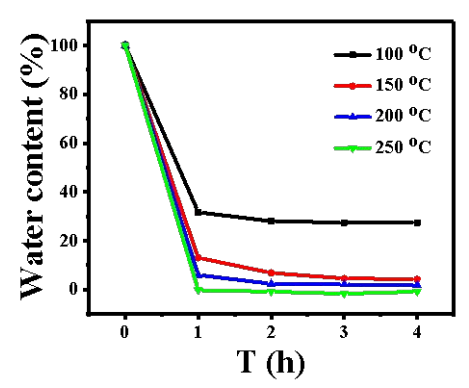

b

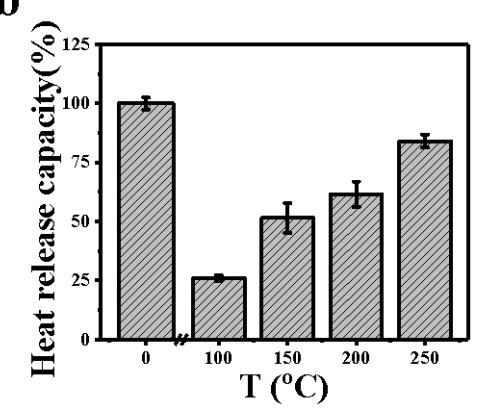

Figure S3. (a) The curve of thermal dehydration of zeolite at different temperature; (b) The heat release capacity of zeolite. 


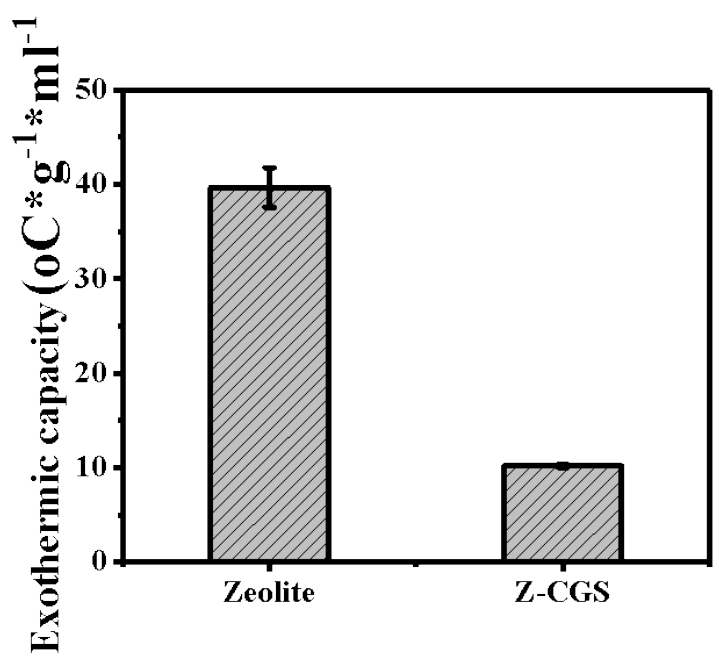

Figure S4. The exothermic capacity of zeolite and Z-CGS. 


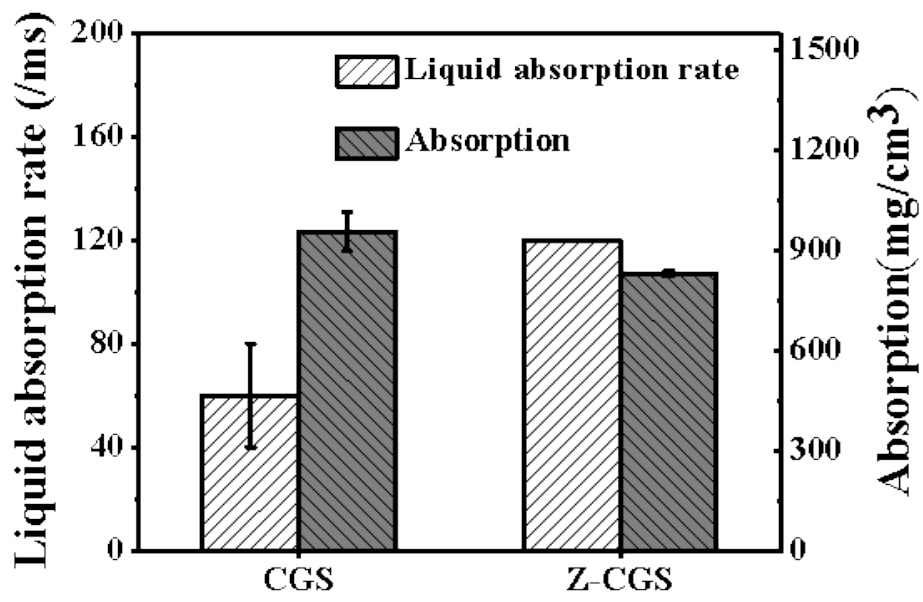

Figure S5. The liquid absorbability for CGS and Z-CGS. 


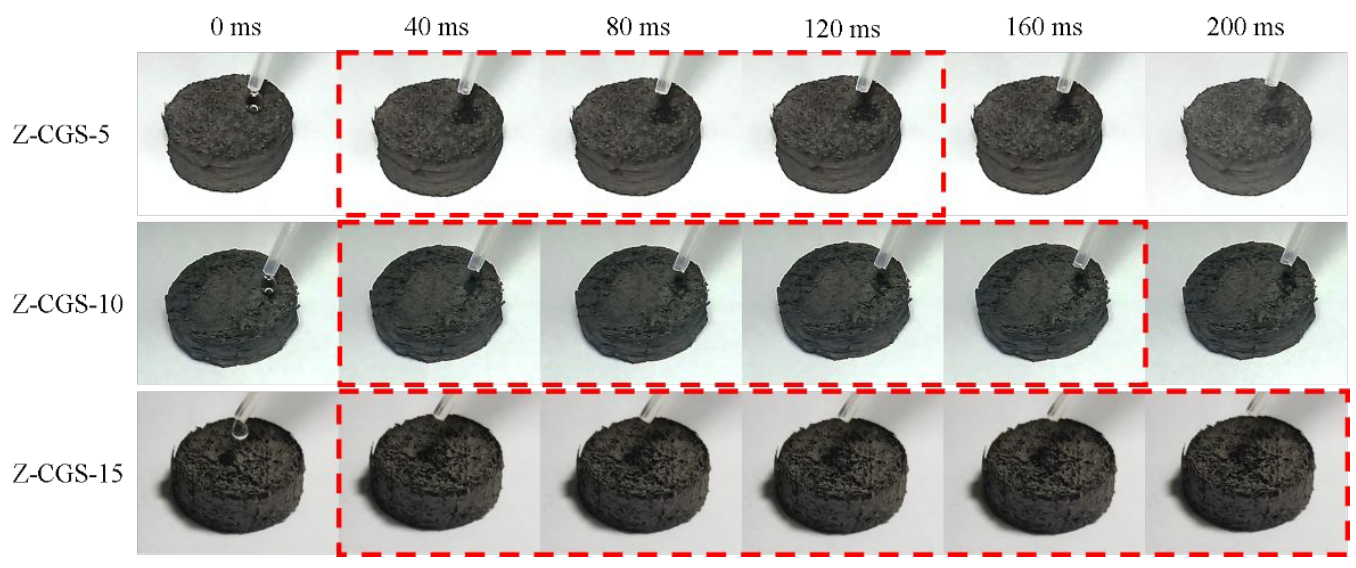

Figure S6. The liquid absorption rate for Z-CGS-5 $(5 \mathrm{mg} / \mathrm{mL})$, Z-CGS $(10 \mathrm{mg} / \mathrm{mL})$ and Z-CGS-10 (15 $\mathrm{mg} / \mathrm{mL}$ ). 


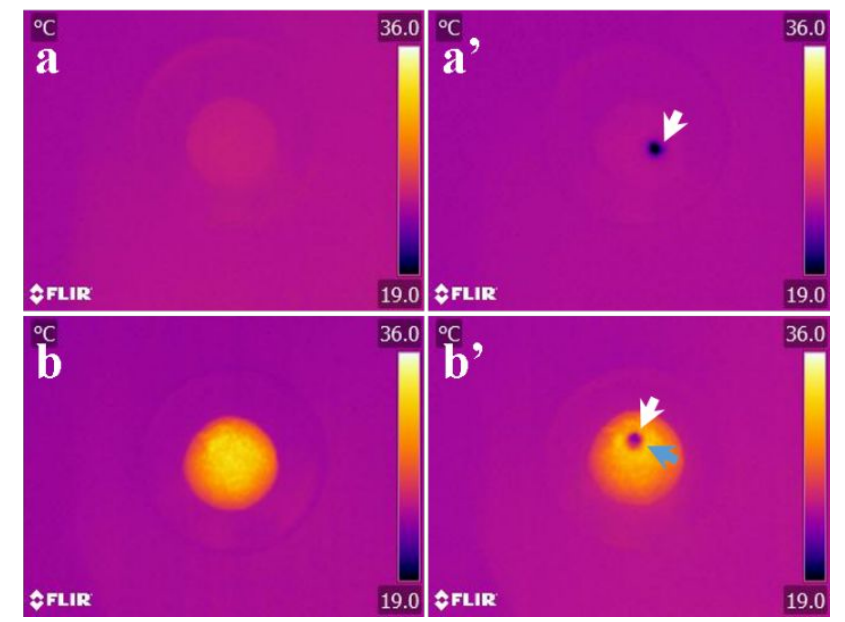

Figure S7. The IR image of Z-CGS. (a and a') $5 \mathrm{mg} / \mathrm{mL}$; (b and b') $10 \mathrm{mg} / \mathrm{mL}$. (a) and (b) Before water absorption; (a') and (b') after water absorption. The situation of liquid drop is marked with white arrow, the situation of warming area is marked with a blue arrow. 


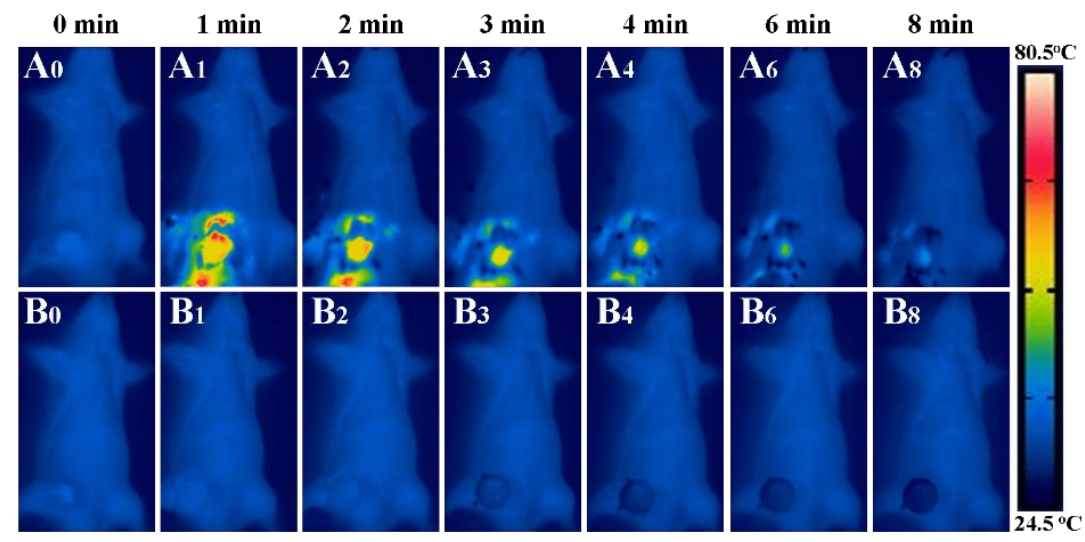

Figure S8. IR images of hemostatic process in the SD rat artery injury model, (A) the zeolite and (B) the Z-CGS, the subscripts shows the time since hemostat applied to wound. 


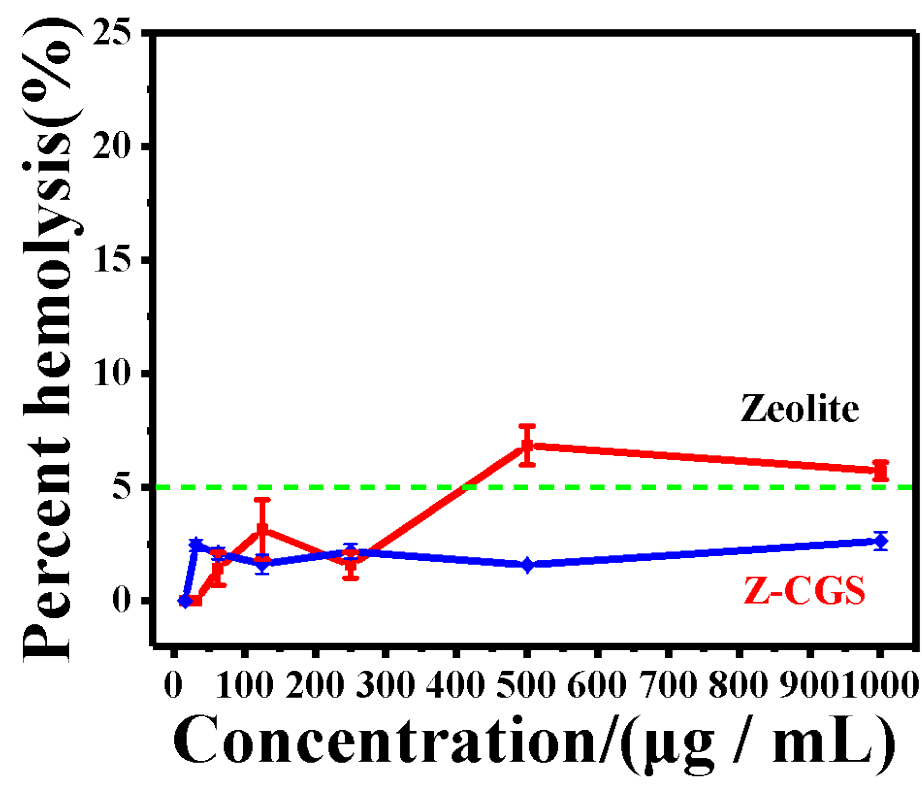

Figure S9. Hemolysis rate of Zeolite and Z-CGS, (5\% is the minimum concentration required for the occurrence of hemolysis). 

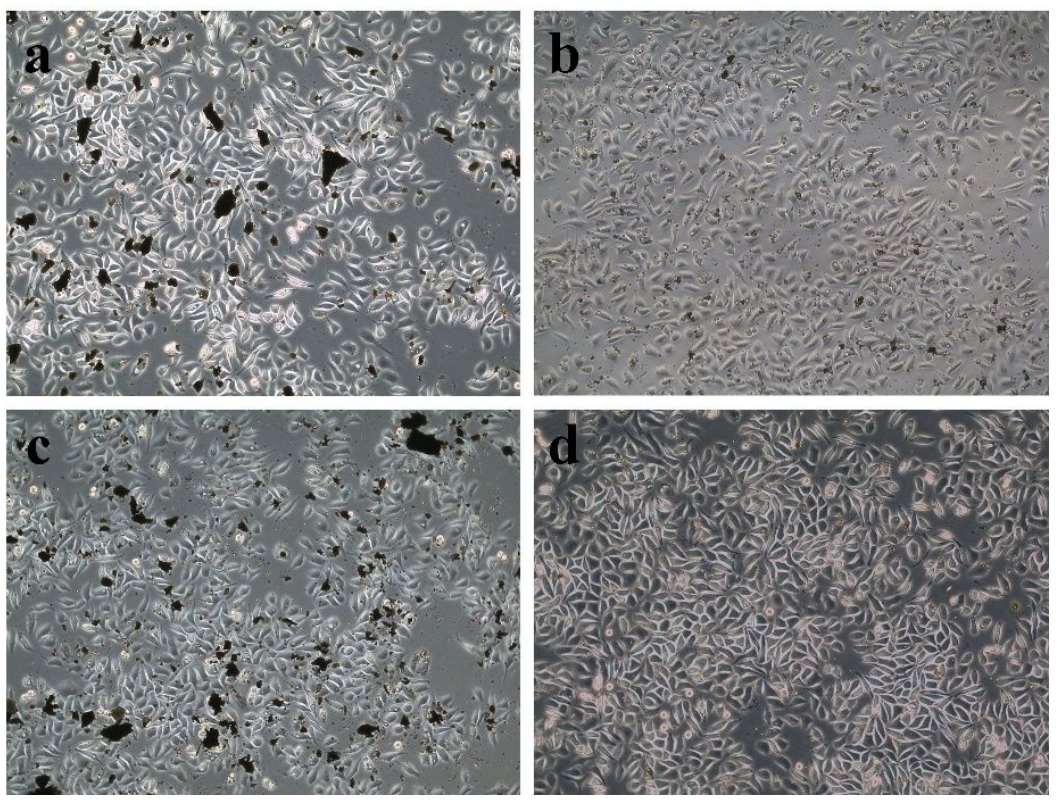

Figure S10. L929 cells cultured with Z-CGS treatments (A, B) and zeolite treatments (C, D). (A, B) Dosage is $62.5 \mathrm{~g} \mathrm{~mL}^{-1}$; $(\mathrm{C}, \mathrm{D})$ dosage is $250 \mathrm{~g} \mathrm{~mL}^{-1}$. 